\title{
RESPONSABILITÉ ÉCONOMIQUE ET SOCIALE DES CONCEPTEURS DE SYSTĖMES D'INFORMATION : CONTRIBUTION À UNE ÉTHIQUE APPLIQUÉE
}

Maryse SALLES

IRIT (SIG/EVI)

Université de Toulouse (UT1-C) Maryse.Salles@ut-capitole.fr

Si les technologies de l'information et leurs multiples applications donnent lieu à une littérature pléthorique, peu de textes sont consacrés à la responsabilité de ceux qui les conçoivent. L'objet de cet article est de traiter de la responsabilité économique et sociale des concepteurs de systèmes d'information (SI), dans le cadre de l'usage professionnel de ceuxci. Par responsabilité, nous entendrons ici, exclusivement, responsabilité morale, c'est-à-dire la nécessité pour une personne de répondre de ses actes devant sa propre conscience, par rapport à ses propres valeurs éthiques. Au sein de cette responsabilité morale, il sera ici question plus spécifiquement des dimensions économique et sociale, au sens où les SI ont un impact sur le développement (et la pérennité) des organisations, et sur les conditions dans lesquelles leurs salariés réalisent leur mission.

Cette question, très peu évoquée dans la littérature du domaine des SI, appartient au champ jeune et encore en formation de l'éthique informatique. La première partie de cet article sera consacrée à un état de l'art de l'éthique informatique (ou éthique des SI). Dans cette littérature, seront en particulier recherchés les outils méthodologiques (ou les éléments permettant de concevoir de tels outils) dont pourraient disposer les concepteurs pour être assistés dans une démarche éthique.

Dans une deuxième partie, nous présenterons notre propre approche de la responsabilité économique et sociale des concepteurs de SI au sein des organisations, en tentant d'éclairer certaines des questions laissées ouvertes dans la littérature d'éthique informatique, et en concluant sur la nécessité de penser une véritable ingénierie de la responsabilité. 
Enfin, dans une troisième et dernière partie, nous proposerons une contribution à une telle ingénierie, sous la forme d'un modèle à trois niveaux, qui permet de révéler comment une vision générale d'une entité (un objet comme l'entreprise, ou une catégorie, comme le travail), et les valeurs éthiques qui l'accompagnent, s'incarnent progressivement dans des principes structurants, puis des normes contraignantes. À titre d'illustration, cet outil méthodologique est appliqué à la catégorie de l'évaluation, pour analyser comment la valeur de démocratie est respectée, ou, au contraire, empêchée.

\section{ÉTAT DE L'ART EN ÉTHIQUE INFORMATIQUE}

Cette section est consacrée à un état de l'art de l'éthique informatique. Après un bref historique, nous présenterons les grandes théories éthiques auxquelles il est fait le plus fréquemment référence, puis nous analyserons les différentes thématiques des textes du domaine.

\section{Bref historique}

Une préoccupation éthique s'exprime dès l'arrivée des premiers ordinateurs, au travers des travaux de Norbert Wiener, qui perçoit dès 1948 les effets potentiels considérables au niveau social de ce qu'il désigne alors sous le terme de "cybernétique »: "Long before Nagasaki and the public awareness of the atomic bomb, it had occurred to me that we were here in the presence of another social potentiality of unheard-of importance for good and for evil ».

Norbert Wiener adopte alors une position très politique. S'alarmant des usages possibles de la cybernétique comme instrument de contrôle par certains groupes ( « Les fascistes, les puissants du monde des affaires, et le gouvernement $")^{1}$, il consacre son ouvrage The Human Use of Human Beings: Cybernetics and Society (1989, orig. 1950) "à une protestation contre cette utilisation inhumaine des êtres humains $»^{2}$.

Dans les années soixante-dix, Mowshowitz et Weizenbaum développeront également un discours critique quant aux possibles impacts sociaux de l'informatique. Mowshowitz (1976) considère ainsi : « Most computer-based information processing systems (...) are seen to serve one of two general social functions: the coordination of diversity or the control of disorder. Coordination and control signify the extremes of a continuum of social choices ».

1. Fascists, Strong Men in Business, and Government.

2. "to a protest against this inhuman use of human beings". 
Il défend la nécessité d'organiser la vigilance pour éviter une dérive vers le pôle «contrôle » de ce continuum. Pour sa part, Weizenbaum (1976) s'attache à définir les domaines dans lesquels les applications informatiques ne devraient jamais être utilisées. Il pose une frontière éthique infranchissable entre le vivant et les systèmes informatiques, et, par ailleurs, exclut de l'informatisation toutes les fonctions humaines qui impliquent des relations interpersonnelles.

Si on s'accorde à considérer Wiener comme le fondateur de l'éthique liée aux systèmes informatiques, c'est Maner qui le premier, en 1978, utilise le terme computer ethics. Il justifie la nécessité d'une éthique propre en soulignant le caractère spécifique des problèmes rencontrés dans l'utilisation des systèmes informatiques.

À partir des années quatre-vingt, l'éthique informatique se constitue en champ de recherche à part entière. Elle devient pluridisciplinaire, accueillant notamment des chercheurs en philosophie (Moor, 1985 ; Johnson, 1985 ; Bynum, 1985). Pour Moor, la technologie informatique est plastique (malleable) au niveau logique, et donc potentiellement universellement applicable. De ce fait, elle crée des situations pour lesquelles nous n'avons pas de règle de conduite prédéfinie (Moor emploie le terme policy vacuum). Les débats philosophiques deviennent dominants dans la recherche du domaine. À titre d'exemple, le caractère unique des problèmes d'éthique informatique défendu par Maner (1978) sera remis en cause par plusieurs auteurs. Johnson (1985) considère ainsi que l'informatique "génère des nouvelles variantes pour des problèmes et dilemmes moraux classiques " ${ }^{3}$, mais ne crée pas de problèmes nouveaux. L'éthique informatique restera durablement marquée par cette orientation philosophique, dont les apports peuvent paraitre aux spécialistes des SI abstraits voire abscons, et difficilement applicables dans la pratique.

Dans les années quatre-vingt-dix, une branche de l'éthique informatique voit le jour, celle de l'éthique professionnelle, qui plaide pour l'intégration de l'éthique dans les cursus de formation des développeurs et dans les méthodes de développement des SI (Gotterbarn, 1991 ; Walsham, 1993). De leur côté, des organisations professionnelles élaborent des codes de bonne conduite, pour l'essentiel aux États-Unis. Ces codes sont de qualité et de portée diverses, depuis les fameux et très discutables Ten Commandments of Computer Ethics de Barquin (1992), le Code of Ethics and Professional Conduct de l'ACM ${ }^{4}$ adopté la même année, jusqu'aux multiples chartes produites dans le sillage du développement durable incluant quelques items d'éthique informatique (CIGREF, 2009).

3. "pose new versions of standard moral problems and moral dilemmas".

4. Association for Computing Machinery. 
Au cours des quinze dernières années, l'Internet, ses usages, les capacités de contrôle étendues qu'il offre, etc. ont marqué les débats, tant parmi les spécialistes de l'éthique informatique qu'au sein d'un large public d'usagers, centrant les débats sur les droits des individus (à la confidentialité, à l'accès à l'information,...) et leurs devoirs ( $c f$. la notion de netiquette).

Malgré des enjeux largement reconnus, les questions d'éthiques sont encore aujourd'hui essentiellement traitées en dehors du domaine des SI, et restent très marginales dans les principales publications, comme le notent Mingers et Walsham (2010) : "Despite the massive effects that developments in ICT are having on the world society, there has not been a huge literature on ethics within the mainstream of information systems journals ».

Il est en effet frappant de constater à quel point la question de l'éthique est absente de la recherche sur les SI. Nous donnons ici deux exemples. Dans une des revues les plus centrales du domaine, MIS Quarterly, seuls 14 articles affichent les termes ethics ou responsibility dans leur titre, nombre qui se réduit à 3 si l'on considère seulement les dix dernières années. La situation est comparable pour Decision Support Systems, revue très représentative du domaine des systèmes d'aide à la décision, avec 7 articles, dont 5 sur les dix dernières années. Par ailleurs, notons qu'en France l'enseignement de l'éthique reste largement absent des cursus informatiques.

\section{Les théories éthiques}

De nombreux textes de la littérature en éthique informatique (laquelle, pour l'essentiel, rappelons-le, ne provient pas du domaine des SI) soulignent la nécessité de situer les recherches au regard des grandes théories éthiques. Ces théories peuvent concerner l'individu, ou bien l'organisation dans son ensemble (en particulier l'entreprise).

\section{L'éthique au niveau de l'individu}

Dans le champ de l'éthique personnelle, les deux théories les plus fréquemment évoquées (et opposées) sont le conséquentialisme, et l'éthique kantienne. L'éthique aristotélicienne apparaît également, de façon plus limitée. Pour le conséquentialisme (en particulier l'utilitarisme de Bentham et Mill), seules les conséquences d'une action peuvent permettre de juger de son caractère éthique. L'impératif moral est d'agir en recherchant la maximisation du bien pour le plus grand nombre.

Dans l'éthique kantienne, les conséquences de l'action ne peuvent permettre d'en qualifier la valeur morale. C'est une éthique du devoir (une déontologie au sens philosophique). Le critère de l'action morale est qu'elle 
puisse être universalisable, ce qui s'exprime dans l'impératif catégorique (Kant ${ }^{5}$, cité par Ricœur, 1998) « Agis uniquement d'après la maxime qui fait que tu peux vouloir en même temps qu'elle devienne loi universelle », complété d'un second impératif (ibid.), "Agis toujours de telle façon que tu traites l'humanité dans ta propre personne et dans celle d'autrui, non pas seulement comme un moyen, mais toujours aussi comme une fin en soi $»$.

Pour Aristote $^{6}$, la vie bonne est le but de la vie. L'éthique est assimilable à la recherche du bonheur (eudaimonia), lequel ne peut s'atteindre que par le développement des vertus. L'action morale est donc celle qui va rendre l'individu meilleur, et c'est à l'aide de ses vertus (en particulier la capacité de choisir le juste milieu, la phronesis) qu'il pourra distinguer les actions morales de celles qui ne le sont pas.

Rappelons que les théories de l'éthique sont très nombreuses et ont donné lieu à d'innombrables débats depuis trois millénaires. Nous évoquerons plus loin les travaux de Paul Ricœur.

Comme nous l'avons indiqué plus haut, les chercheurs et professionnels en informatique se sentent souvent étrangers à ces théories (CIGREF, 2013). Stahl (2008a), qui est un philosophe, remarque ainsi que les chercheurs en SI, devant la complexité des débats en éthique, ont la tentation d'ignorer les apports de la philosophie et de travailler à partir de « concept de l'éthique issu du sens commun, où les comportements ou les opinions sont considérés comme éthiques si les personnes concernées les perçoivent comme éthiques » ${ }^{7}$, et il conclut «Une telle approche n'est pas tenable ${ }^{8}$.

On peut, symétriquement, souligner l'effort que les philosophes doivent eux-mêmes produire pour rendre leurs concepts utilisables par les chercheurs et praticiens de l'informatique, nécessité dont certains d'entre eux sont conscients, comme Nissenbaum (1998), lorsqu'elle écrit à propos de valeurs comme la justice, la responsabilité ou l'autonomie : "Out of these conceptions, in order to be able to map values to characteristics of computer systems, I must construct concepts that are operational within a practical setting, create precision where none naturally exists».

\section{L'éthique au niveau de l'entreprise}

L'éthique de l'entreprise vue comme une entité est traitée au travers de l'éthique des affaires (business ethics), qui a inspiré la notion de Responsabilité

5. La Métaphysique des mours (1795).

6. L'éthique à Nicomaque.

7. "Common sense concept of ethics, where behaviors or views are accepted as ethical if respondents perceive them as ethical".

8. "Such an approach is not tenable". 
Sociale de l'Entreprise (Bollecker et al., 2008). L'éthique des affaires a donné lieu à un ensemble de théories que Smith et Hasnas (1999) qualifient de normatives.

Une première approche est celle de la «théorie de l'actionnaire » (stockholder theory), pour laquelle la seule mission de l'entreprise est de faire du profit, notamment pour rémunérer les actionnaires. Le comportement moral de l'entreprise s'inscrit dans cette mission, et les cadres et salariés ont donc pour devoir d'agir afin de maximiser le profit, dans un cadre légal bien entendu. Le représentant le plus connu de cette théorie est Milton Friedman, qui a intitulé l'un de ses articles "The Social Responsability of Business is to Increase its Profit ». La " théorie de l'actionnaire » s'inscrit dans le courant utilitariste, car ce sont les conséquences des actions qui vont déterminer si elles sont éthiques ou non (c'est-à-dire, ici, si elles permettent ou non d'accrôttre le profit).

La théorie des parties prenantes (stakeholder theory) est la deuxième principale approche en éthique des affaires. Bien que pour certains courants de cette théorie, la liste des parties prenantes soit extensive, incluant parfois la société dans son ensemble, les parties prenantes habituellement considérées sont celles qui soit sont vitales pour la réussite de l'entreprise, soit sont vitalement affectées par elle (Smith, Hasnas, 1999) : actionnaires, salariés, fournisseurs, clients, habitants et pouvoirs locaux des zones d'activité de l'entreprise... L'éthique kantienne s'applique ici dans le sens où aucune partie prenante ne doit être considérée uniquement comme un moyen par les dirigeants de l'entreprise, mais doit être respectée comme une fin.

La théorie du contrat social représente une troisième orientation. L'idée de base est que les membres de la société garantissant un certain nombre de droits à l'entreprise (utiliser des ressources naturelles, employer des personnes...), ils attendent en échange d'une part que leur bien-être (en tant que consommateurs, salariés, citoyens...) en soit amélioré, d'autre part que l'entreprise respecte les principes généraux de la justice (Donaldson ${ }^{9}$ cité par Smith et Hasnas, 1999). Cette approche est parfois critiquée, comme l'est la RSE, car elle permettrait à l'entreprise de se poser comme une entité éthique, ayant donc une légitimité à s'opposer à l'existence de régulations qui lui seraient extérieures (régulations nationales ou internationales) (Stahl, 2008b).

\section{Les thématiques de l'éthique informatique}

L'éthique informatique est un domaine récent, dont les thématiques peinent encore à s'inscrire dans une structure générale. Nous proposons dans

9. Donaldson, T. J. (1982), Corporations and Morality, Prentice-Hall, Englewood Cliffs, NJ. 
cette section une organisation du champ autour des questions suivantes : les valeurs éthiques, les types d'agents concernés et leurs rapports à l'informatique (usagers ou concepteurs), les usages de l'informatique, et, en particulier l'aide à la décision, pour finir sur la question de la construction des SI et de l'intégration de l'éthique dans les méthodes de conception.

La question des usages illégaux ne sera pas traitée ici, car ne relevant pas selon nous de l'éthique mais du droit. Nous considérons en effet que dans le cas d'organisations (publiques ou privées), le principe éthique général du respect de la loi peut être considéré comme intégré dans le fonctionnement.

\section{Valeurs éthiques}

Certains auteurs limitent la liste des valeurs de l'éthique informatique à quatre thèmes (Mason, 1986) connus sous l'acronyme PAPA : privacy (respect de la vie privée), accuracy (précision et fiabilité de l'information), property (le respect de la propriété), accessibility (garantie de l'accès à l'information). Mais depuis quelques années, de nouvelles valeurs sont fréquemment citées comme la justice (qui recouvre le concept de fairness, et celui de non-discrimination), la liberté (de parole, d'accès à l'information), l'autonomie de l'utilisateur, la neutralité (freedom from bias), la transparence, la confiance, le consentement éclairé (informed consent), l'imputabilité (accountability) qui permet d'établir un lien entre une action ou une information et son auteur, l'utilisabilité pour tous (universal usability), le bien-être humain (human welfare), la démocratie (Friedman et al., 2002 ; Brey, 2010 ; Floridi, 2008, 2010). Des valeurs moins spontanément attachées à la notion d'éthique sont parfois ajoutées à cette liste, comme la courtoisie, le calme (calmness), l'identité (Friedman et al., 2008).

Le développement durable est également inclus dans les valeurs éthiques, et peut même représenter l'unique contexte dans lequel l'éthique informatique est abordée par une organisation professionnelle (CIGREF, 2009).

Le concept de neutralité, lié à celui de biais (bias), est en général employé pour faire référence au risque que certains groupes soient lésés par le système informatique (par exemple par le principe de classement des résultats d'une requête, ou par une représentation inégalitaire des différents groupes). Mais il est aussi utilisé en rapport avec la notion de vérité, qu'il ne serait pas éthique de tronquer, de masquer ou de dénaturer.

Notons par ailleurs que lorsque la responsabilité est évoquée, c'est assez rarement de responsabilité sociale qu'il s'agit, et pratiquement jamais de responsabilité économique. La responsabilité est en général liée à la notion d'imputabilité (accountability). Or celle-ci est assez restrictive et principalement 
orientée vers les usages incivils ou frauduleux, dont il est souhaitable de pouvoir identifier les auteurs.

\section{Utilisateurs ou concepteurs}

Les premiers travaux en éthique informatique portaient sur les usages de l'informatique et leurs conséquences, les transformations que ces usages apportaient dans la société, les dangers nouveaux qui les accompagnaient. L'extension considérable d'Internet et du nombre de ses utilisateurs a accentué cette tendance, entraînant l'éthique informatique vers des analyses sociétales.

Un autre courant s'est cependant développé, en particulier depuis la toute fin du siècle dernier, qui s'intéresse à la conception de systèmes informatiques, non pas en proposant des listes de valeurs que les concepteurs se devraient de respecter, mais en fournissant des méthodes de conception éthiques (voir plus bas).

S'il est bien entendu qu'il n'y a pas de frontière étanche entre l'éthique considérée du point de vue des utilisateurs des systèmes informatiques et celle destinée aux concepteurs de ces systèmes, rappelons que notre travail se focalise sur ces derniers.

\section{Types d'usages}

Un grand nombre de travaux est consacré aux usages personnels (et non professionnels) de l'informatique, en particulier des applications disponibles au travers d'Internet. Quand les usages professionnels sont évoqués, c'est en général de façon très globale, et non discriminante. Les valeurs éthiques considérées ne diffèrent alors pas d'un type d'usage à l'autre : il faut respecter la vie privée des salariés, ne pas établir de discrimination entre groupes de salariés, les salariés ne doivent pas envoyer des mails injurieux à leurs collègues, etc.

Quelques auteurs abordent les conséquences sociales des SI, notamment sur la nature du travail (Stahl, 2010). Notons qu'au sein des usages professionnels, il n'est pas réellement établi de distinction entre l'usage de l'informatique comme aide à la réalisation d'une tâche, et son utilisation en tant qu'aide à la décision.

\section{L’aide à la décision}

Si l'éthique dans la prise de décision a donné lieu à une importante littérature en médecine, et dans le domaine des sciences de gestion, notamment pour les activités de conseil (Cottone, Claus, 2000), l'éthique des systèmes d'aide à la décision reste un domaine peu exploré. 
Après n'avoir trouvé qu'un nombre extrêmement réduit d'articles sur le thème de l'éthique dans deux revues majeures du domaine de l'aide à la décision (Decision Support Systems et Decision Sciences), Meredith et Arnott (2003) remarquent: " Given the popularity of data warehouse, business intelligence and other decision support systems, it is unfortunate that the ethics of decision support as a specific topic has received very little attention in comparison to the issues of privacy and other general IT ethics issues ".

Deux approches de l'éthique en aide à la décision peuvent cependant être distinguées.

La première approche considère les conséquences des décisions prises à l'aide d'un système d'information d'aide à la décision (SIAD), et suggère que les décideurs devraient être alertés sur ces conséquences par le SIAD même. Mathiesson (2007) propose de concevoir des SIAD éthiques (ethical decision support systems ou EDSS) et décrit les caractéristiques que devraient posséder de tels systèmes. Un EDSS devrait ainsi ne pas guider le décideur, mais plutôt lui offrir un ensemble d'outils lui permettant d'approcher au mieux sa propre exigence éthique (dont on lui fait le crédit).

Dans la même perspective, et faisant le constat que les décisions prises à l'aide de SIAD peuvent affecter un grand nombre de parties prenantes, en particulier dans le cas de la décision publique, Chae et al. (2005) défendent l'idée que les concepteurs de SIAD ne doivent pas considérer uniquement les facteurs techniques, mais aussi les considérations éthiques et morales. La seconde approche s'intéresse à l'influence que peut avoir le SIAD sur la façon dont la décision est prise (le processus de prise de décision). Nous reviendrons plus loin sur cette question.

\section{Développement des SI}

Comme nous l'avons vu plus haut, il existe une certaine distance (voire un fossé) entre les productions des chercheurs en éthique informatique originaires des sciences humaines ou sociales et les pratiques des chercheurs et professionnels de l'informatique. Cette question a été évoquée de façon récurrente par les deux parties (Rogerson et al., 2000 ; Nissenbaum, 1998 ; Bell, Adam, 2004). Van Den Hoven (2008) plaide ainsi pour « une intégration proactive de l'éthique (...) dans la conception, l'architecture, les exigences, les spécifications, les standards, les protocoles, les systèmes d'incitation et les arrangements institutionnels $»^{10}$.

10. "A proactive integration of ethics (...) in design, architecture, requirements, specifications, standards, protocols, incentive structures, and institutional arrangements". 
Depuis une quinzaine d'années, un courant de recherche se centre sur l'applicabilité de l'éthique informatique dans le cadre du développement d'un système. Plusieurs démarches ont été proposées pour inclure une perspective éthique dans la conception des SI. Ces approches ont été majoritairement produites par des chercheurs en sciences humaines ou sociales, souvent en coopération avec des chercheurs en SI. Elles sont de niveaux variés quant à leur exhaustivité, leur degré d'opérationnalité et le nombre de leurs cas d'application.

Certaines de ces démarches cherchent à accompagner du mieux possible l'organisation des échanges et négociations entre les différentes parties prenantes. Mingers et Walsham (2010) proposent ainsi de s'appuyer sur l'éthique du discours d'Habermas ${ }^{11}$ (1992, cité par les auteurs) afin de donner aux diverses parties prenantes des droits égaux, et de parvenir à un consensus dans les meilleures conditions possibles. Mingers et Walsham suggèrent que des méthodes de développement de type $\mathrm{SSM}^{12}$ comme $\mathrm{JAD}^{13}$ peuvent être utilisées dans cette perspective.

La méthode Value Sensitive Design (VSD) est l'une des réalisations les plus abouties en matière d'intégration des dimensions éthiques dans le développement des SI (Friedman et al., 2002). À l'origine de VSD on trouve le concept de valeurs intégrées dans les SI (embedded values) (Nissenbaum, 1998). Ces valeurs sont encodées dans les systèmes sans que les concepteurs de ces systèmes en aient toujours conscience. Invisibles, elles orientent pourtant le fonctionnement du SI, et peuvent être en contradiction avec les valeurs éthiques des utilisateurs. Un exemple donné est celui des cookies qui permettent à des sites de collecter de l'information sur une personne connectée, sans que celle-ci en soit informée ni, a fortiori, n'ait donné son consentement, en opposition avec la valeur éthique de consentement informé.

VSD est une méthodologie itérative qui intègre trois types d'analyses : conceptuelle, empirique et technique. L'analyse conceptuelle cherche principalement à déterminer quelles sont les parties prenantes directement ou indirectement impactées par le système à développer et la manière dont elles le sont, puis à identifier les valeurs impliquées, enfin à construire des définitions précises pour ces valeurs. L'analyse empirique étudie le contexte humain et social, individuel et organisationnel, dans lequel le système doit fonctionner. La dernière analyse, l'analyse technique, d'une part cherche à évaluer si les solutions techniques envisagées peuvent constituer une aide, ou au

11. Habermas, J. (1999), A genealogical analysis of the cognitive content of morality, in Habermas, J. (ed.), The Inclusion of the Other, Cambridge, Polity Press, 3-48.

12. Soft Systems Methodologies.

13. Joint Application Design. 
contraire un obstacle à la prise en compte des valeurs retenues, d'autre part propose des développements spécifiques pour soutenir telle ou telle valeur.

Parmi les nombreux logiciels développés en intégrant l'approche VSD, on peut citer UrbanSim (Borning et al., 2007). Il s'agit d'un système d'aide à la décision en aménagement du territoire, destiné à un large ensemble de parties prenantes (élus, habitants, aménageurs...), qui permet de visualiser les résultats de différents scénarios d'évolution. Les valeurs morales soutenues par UrbanSim sont la justice (non-discrimination d'un groupe de parties prenantes), l'imputabilité (les parties prenantes doivent être à même de vérifier que leurs valeurs sont bien traduites dans le système), et la démocratie (le système soutient le débat démocratique).

Dans la perspective de VSD, Brey (2008) introduit une distinction entre deux types pratiques de développement, de gestion ou d'utilisation de SI. Il nomme pratiques moralement transparentes celles qui sont en relation (positive ou négative) avec des valeurs éthiques manifestes. Il qualifie d'opaques des pratiques mal connues au-delà d'un cercle restreint de spécialistes et qui ont un impact quant à des valeurs morales, ou des pratiques connues, mais dont on ne comprend pas aisément qu'elles portent une charge éthique. Brey propose une démarche qu'il nomme disclosive computer ethics (que l'on pourrait traduire par " éthique informatique de divulgation »), centrée sur l'identification des pratiques moralement opaques.

\section{Conclusion sur l'éthique informatique}

En conclusion de cette section, on peut souligner que l'éthique informatique reste un domaine encore en formation, dont le caractère pluridisciplinaire rend la structuration complexe. Il connaît des évolutions positives, qui tendent à réduire la distance entre chercheurs en sciences humaines et sociales et chercheurs et praticiens en informatique, notamment au travers de l'effort produit par les premiers pour offrir aux seconds des outils opérationnels.

Plusieurs questions restent cependant selon nous à explorer ou approfondir. Les valeurs éthiques sont souvent présentées sous forme de listes simples, sans catégorisation ni hiérarchie. De même, les relations de dépendance entre valeurs éthiques sont très peu explorées. Il serait intéressant d'étudier certaines de ces relations : relation qui associerait certaines valeurs à certaines visions du monde, relation qui permettrait d'identifier des valeurs génériques (dont la présence dans un système entraînerait « automatiquement » la présence d'un ensemble de valeurs d'un niveau inférieur), relation d'opposition qui alerterait sur le fait que la présence simultanée de deux valeurs est difficile à assurer, etc. 
L'absence d'une cartographie des valeurs éthiques est à rapprocher de la remarque de Stahl (2008a, 2008b), qui constate que beaucoup de travaux en éthique informatique se situent au niveau micro, en considérant le niveau macro comme donné. Le niveau macro est pour l'auteur le niveau ontologique, celui où l'on précise comment on considère un certain nombre d'entités essentielles. Au niveau macro, on pourra par exemple s'interroger sur la vision à adopter pour l'homme : un être recherchant à maximiser son profit individuel, ou à l'inverse un être recherchant une harmonie sociale ; pour l'entreprise : entité ayant comme raison d'être la maximisation du profit pour les actionnaires, ou institution qui doit produire des biens ou services utiles à la société (le profit étant alors un objectif et non la raison d'être).

Une conséquence de ce positionnement au seul niveau micro est que l'origine des valeurs (éthiques ou " anti-éthiques ») présentes au final dans les SI n'est que très peu interrogée. Il en est de même pour l'analyse des obstacles à l'intégration de valeurs éthiques dans les SI, ou à la suppression de valeurs « anti-éthiques».

En outre, une analyse des rapports entre les SI et les organisations qui les produisent et/ou qui les utilisent (en particulier les entreprises), absente de la littérature, permettrait sans doute de mieux approcher ce que sont les valeurs intégrées (embedded values). Il serait également nécessaire de déterminer les moments et modes les plus fréquents de leur inclusion dans les systèmes. Quelles phases du processus de conception d'un système, quels types de méthodes, quels types d'outils de développement, quels types progiciels ( $\mathrm{PGI}^{14}$ par exemple), etc., sont-ils des voies privilégiées par lesquelles certaines valeurs sont intégrées dans les SI ? Notons que très peu de textes traitent de la nature des SI, ou de leurs différentes fonctions au sein des organisations.

En lien probable avec ce qui précède, on peut noter que les méthodes d'éthique informatique accompagnent le développement du système informatique dans un séquencement qui leur est propre, sans véritablement s'insérer dans les différentes étapes de la conception. Dans la méthode VSD par exemple, l'analyse technique est focalisée sur le développement, et considère peu la conception, c'est-à-dire la phase où sont produites les modélisations de données et de traitements, lesquelles sont pourtant très structurantes du SI produit.

Sur un autre plan, notons que dans les travaux d'éthique informatique, les SI internes aux entreprises sont considérés de façon non différenciée par rapport aux applications qui touchent le grand public. Or, les premiers nous

14. Progiciels de Gestion Intégrée, en anglais Enterprise Resource Planning (ERP). 
semblent mériter un traitement spécifique, de par leur impact économique et leurs caractéristiques propres, dont nous traiterons dans la section suivante.

Ce manque d'intérêt pour les SI internes aux organisations s'accompagne d'une certaine indifférence envers les rapports de pouvoir qui peuvent exister dans ces organisations, à quelques notables exceptions comme les travaux pionniers de Wiener (1948) et Mowshowitz (1976), ou, plus récemment, Walsham (1993, 2011). Les différentes parties prenantes sont en général considérées comme disposant de capacités d'intervention et d'influence égales.

Dans la même perspective, il est admis qu'il existe bien une vérité unique, exogène aux organisations, immanente et donc commune à toutes les parties prenantes, et qui sert d'étalon à la valeur de neutralité (freedom from bias).

Enfin, et ceci nous paraît être une conséquence de l'ensemble des points qui précèdent, la question du sens produit par et dans les SI (vision du monde ou sémantique qui s'impose à leurs utilisateurs et structure leur comportement cognitif), n'est abordée que de façon extrêmement restrictive (essentiellement au travers des questions de discrimination), à l'exception de certains travaux dans le domaine de l'aide à la décision (Meredith, Arnott, 2003).

\section{PROBLÉMATIQUE ET POSITIONNEMENT DE LA RECHERCHE}

L'objet de cette section est de préciser ce qui fonde notre approche de la responsabilité économique et sociale des concepteurs de SI au sein des organisations, dans une perspective d'éthique appliquée. Nous essaierons ce faisant d'éclairer certains des points qui viennent d'être évoqués.

Dans un premier temps, nous définirons ce qu'est un SI, ses fonctions au sein d'une organisation et ses caractéristiques, en particulier celles qui sont susceptibles de générer des valeurs intégrées (en accord ou en opposition avec des valeurs éthiques). Seront ensuite présentés notre posture de recherche, puis les objectifs poursuivis par ce travail.

\section{Systèmes d'information, systèmes informatiques, systèmes d'aide à la décision}

«Système d'information » est un concept issu de la systémique. Le système d'information, qui dépasse et englobe le système informatique, est avant tout vu comme un système de représentations. Ces représentations portent sur 
l'organisation elle-même (ses activités, ses acteurs, ses objets, les façons de les catégoriser, les relations qui les lient, les types d'actions qu'ils effectuent ou qu'ils subissent, les procédures...), mais aussi sur son environnement et les relations qu'elle entretient avec lui.

Le système d'information remplit deux fonctions principales (Le Moigne, 1977) : la première est d'exprimer les représentations partagées indispensables à la réalisation de la mission (raison d'être) du système, c'est-à-dire à la production au sens large ; la seconde est de produire les représentations du système et de son environnement nécessaires au pilotage.

Le système informatique (que nous abrégeons ici en SI), sous-ensemble du système d'information, assure la part automatisable de ces deux fonctions, en encodant les représentations à l'aide de la technologie informatique. À l'origine très centrés sur l'assistance à la réalisation des tâches, les SI, à partir des années quatre-vingt, se sont étendus vers l'aide à la décision, au travers des systèmes d'aide à la décision.

\section{Les SI comme systèmes performatifs}

Un trait marquant des systèmes d'information, encore renforcé dans les systèmes informatiques, est leur caractère normatif, et, au-delà performatif ${ }^{15}$.

Ce qui est défini dans le système d'information acquiert ainsi un statut de «réalité» et permet, voire entraîne systématiquement, la décision et l'action. Ainsi, certaines affections, quand elles apparaissent dans une nomenclature officielle, entraînent une offre pharmaceutique spécifique ( $c f$. les débats récents autour de la nouvelle nomenclature des troubles mentaux aux ÉtatsUnis, dont il a été dit qu'elle créait des maladies mentales ${ }^{16}$ ). Nous reviendrons plus loin sur un cas intéressant (et extrême) de cet effet performatif, celui des indicateurs de gestion. Comme l'écrit Desrosières (2003) à propos de la statistique, les catégories, les objets de la comptabilité publique ou privée, les référentiels, les indicateurs,... ne sont pas de simples reflets de la réalité, mais «en revanche, il[s] contribue[nt] à la façonner, à la transformer, sinon à la créer».

À l'inverse, ce qui n'est pas présent dans le système d'information peine à exister et à faire l'objet de décisions ou d'actions. Les SI des entreprises qui ont perdu des compétences importantes lors du départ à la retraite de certains salariés n'incluaient sans doute pas d'information sur ces compétences. Au plan d'une collectivité territoriale, des nouvelles activités non encore

15. Nous employons ici ce terme dans un sens plus large que celui proposé à l'origine par Austin, et l'entendons comme une capacité à imposer, par le langage, l'existence d'un objet.

16. Un article du Monde du 13/05/2013 titrait «Psychiatrie : DSM-5, le manuel qui rend fou ». 
recensées par l'INSEE (comme les systèmes embarqués, ou les éco-industries) feront difficilement l'objet de politiques spécifiques, car il est impossible d'identifier les entreprises de ces secteurs sur la base de leurs codes APE.

L'informatique a un rôle amplificateur de la performativité, d'une part parce que cette technologie fonctionne sur la base de modélisations, qui sont autant de réductions de la complexité, d'autre part parce que, pour un très grand nombre d'activités, les systèmes informatiques représentent l'interface obligatoire entre les salariés et le monde (et souvent le seul accès à celui-ci). Ceci concerne notamment, de façon de plus en plus marquée, la prise de décision. Meredith et Arnott (2003) considèrent ainsi que les SIAD limitent l'indépendance des décideurs, en imposant des structures à leur pensée, en dirigeant leur attention vers telle ou telle information :

"Another distinguishing feature of DSS [decision support systems] development and use is the impact of the system upon the cognitive strategies and structures of the user. Whilst an operational system has some impact upon its users in terms of understanding and task approach, the degree to which a DSS has an impact on the user's cognitive strategies and structures is much greater due to the uncertain, unstructured nature of the task».

Notons que les décisions non structurées (tactiques et stratégiques) dont il est question dans cette citation sont celles qui ont les impacts les plus importants sur l'organisation. Ces décisions sont celles qui sont en lien avec l'innovation (qui concerne tous les secteurs de l'entreprise : définition des produits, procédés de fabrication, segmentation de marché, méthodes, organisation, système d'information, etc.). L'innovation n'est possible que si l'entreprise a la capacité de générer des représentations innovantes (concernant ses compétences, ses marchés, ses produits, son environnement économique...). Rappelons enfin que l'innovation est aujourd'hui considérée comme le premier facteur de compétitivité des entreprises (Moati, 2008). La situation est très comparable pour les institutions publiques. Pour assurer un développement économique pérenne, une collectivité territoriale doit mettre en œuvre des représentations innovantes de ce qu'est un territoire, une ressource, un acteur économique, une filière industrielle, etc.

L'un des rôles clés des SI, et singulièrement des SIAD, doit donc être d'aider à construire ces représentations innovantes. La responsabilité économique du concepteur est de soutenir la création de ces représentations, ou, au minimum, de ne pas l'entraver en codant dans le SI une vision unique de l'organisation et de son environnement (Salles, 2013). 


\section{Les SI comme dispositifs de pouvoir}

Plusieurs auteurs, appartenant à diverses disciplines, analysent les systèmes informatiques en termes de dispositifs de pouvoir au sein des organisations.

Dans le domaine de l'éthique informatique, Walsham (2011) souligne ainsi que les concepteurs de SI sont impliqués dans la production et la reproduction de valeurs normatives et dans le renforcement ou la modification de rapports de pouvoir, et conclut « les responsables de la stratégie du SI sont ainsi impliqués dans des choix moraux concernant des normes et des relations de pouvoir, même si ces choix ne reflètent aucune intention consciente de leur part $»^{17}$.

Dans le même esprit, Kvasny et Richardson (2006) plaident vigoureusement pour une recherche critique dans le domaine des SI en citant un ensemble de questions sociales liées à l'usage de l'informatique dans les organisations. Hall et Stahl (2012) montrent comment certaines technologies informatiques émergentes accompagnent une marchandisation généralisée de la société.

En France, la sociologie de la gestion s'intéresse aux dispositifs de gestion, parmi lesquels les SI tiennent une place prédominante. Pour Maugeri (2007) les SI organisent le travail, affectent et prescrivent les tâches, contrôlent leur bonne exécution et interviennent dans l'évaluation des personnels, avec une puissance toute particulière : "À cet égard, il faut admettre que l'informatique met à la portée du management des possibilités d'organisation et de contrôle du travail jamais atteintes jusque-là. Occulter cette dimension, c'est s'interdire de voir ce qui a changé dans l'équilibre des forces entre les parties engagées dans le processus de travail. »

Il souligne l'asymétrie, soutenue par le SI, entre les différentes parties prenantes de l'entreprise : "Il est inutile de se cacher derrière la "liberté » de l'acteur pour nier la dimension coercitive de certaines situations de travail. L'action est, dans certains cas et pour certains salariés, bel et bien contrainte. »

Évoquant le rôle des SI dans la gestion publique, Le Galès (2005) montre que la restructuration de l'État au Royaume-Uni n'a été possible que grâce à «l'élaboration d'un SI de très grande ampleur », et évoque une véritable «industrie de l'indicateur».

17. "IS strategists are thus implicated in moral choices regarding norms and power relations, even if this does not reflect any conscious intention on their part". 


\section{Une posture de recherche critique}

Selon la classification de Neuman (2000), et en opposition avec les recherches positiviste et interprétiviste, notre perspective est ici celle de la recherche critique : « [Critical approach] goes beyond surface illusions to reveal underlying structures and conflicts of social relations as a way to empower people to improve the social world». Cette orientation est encore relativement peu présente dans la recherche française sur les SI, comme le note Rowe (2009).

Stahl (2008a) note "[qu']il existe un lien intrinsèque entre éthique et recherche critique ${ }^{18}$, et poursuit en soulignant la nécessité pour les chercheurs critiques de préciser sur quels postulats éthiques ils se fondent.

Pour présenter ces postulats, nous nous appuierons sur Ricœur (1998), qui établit une distinction entre éthique et morale. Il réserve le terme d'éthique "pour tout le questionnement qui précède l'introduction de l'idée de loi morale », et désigne par morale " tout ce qui, dans l'ordre du bien et du mal, se rapporte à des lois, des normes, des impératifs ». Nous retiendrons l'idée d'une chaîne, qui mène des principes éthiques les plus génériques vers les impératifs moraux les plus applicables, ou, selon Ricœur (1998), qui reconstruit « tous les intermédiaires entre la liberté, qui est le point de départ, et la loi, qui est le point d'arrivée».

Au niveau le plus élevé est donc la liberté d'agir, la « capacité de se désigner comme l'auteur véritable de ses propres actes » (Ricœur, 2000). Cette liberté est celle du chercheur, elle est aussi celle du concepteur de SI, que nous considérons responsable (ou co-responsable) du respect de principes éthiques et de normes morales dans le fonctionnement du SI. Elle est, enfin, celles des autres parties prenantes de l'organisation.

\section{Les objectifs de la recherche}

Geoff Walsham a titré l'un de ses récents articles Are We Making a Better World with ICTs?. Notre recherche s'inscrit, modestement mais délibérément, dans la visée d'un monde meilleur. La démocratie est la valeur qui nous semble le mieux à même de servir ce but.

\section{La valeur de démocratie}

La valeur éthique principale que nous cherchons à soutenir est donc celle de démocratie. Cette valeur a pour nous un statut paradigmatique. Nous la plaçons au sommet de la hiérarchie des valeurs éthiques, son respect devant entraîner celui d'une série d'autres valeurs.

18. "there is an intrinsic link between ethics and critical research". 
Il s'agit donc pour nous d'aider à produire des SI, et en particulier des SIAD, qui respecteront des exigences de démocratie. La démocratie est ici considérée avant tout comme la garantie de l'accès à des visions du monde plurielles. Elle n'est donc pas centrée, comme dans la plupart d'approches d'éthique informatique qui en traitent, sur le droit à la parole de groupes ou des individus, même si nous y incluons le principe du respect des intérêts de chacune des parties prenantes.

Aider à une plus grande démocratie dans les organisations est selon nous à même d'aider au développement à long terme de l'organisation, et, plus largement, à un développement réellement durable pour la société. Ceci passe par la lutte contre ce qui, dans les SI des organisations, peut entraver l'innovation, en particulier dans la prise de décision. Cette aide à la démocratie consiste en particulier à découvrir dans ces SI ce qui rend possible d'imposer une vision unique des objets, acteurs, catégories..., utiles à l'organisation.

Pour des institutions publiques, la promotion de la démocratie s'attachera à identifier, dans les instruments de l'action publique, les choix politiques dissimulés au sein des outils informatiques, choix non débattus pas les élus et qui s'imposent à eux (Lascoumes, Le Galès, 2005). Il s'agit donc dans ce cas d'aider à un réinvestissement du politique par les élus.

\section{La nécessité d'outiller la responsabilité des concepteurs de SI}

Le concepteur de SI et de SIAD, nous l'avons dit, doit être « auteur de ses propres actes », et donc responsable. Notre position est que cette capacité d'agir, cette responsabilité (morale aussi bien qu'économique et sociale) doit être assistée. Une telle assistance ne sera selon nous efficace que si elle est intégrée de façon structurelle dans les méthodes de conception de SI.

Notons que nous entendons ici par méthode, non pas une simple démarche, mais un ensemble de quatre composants (Seligmann et al., 1989), désignés comme autant de «manières de » (way of) : 1) la manière de penser (fondements théoriques, paradigme), 2) la manière de modéliser les données, les traitements, les interfaces, 3 ) la manière d'organiser (démarche, processus de la méthode), 4) la manière d'aider à la mise en œuvre de la méthode. Les méthodes d'éthique informatique sont souvent centrées sur une démarche (point 3), parfois accompagnées d'une définition des paradigmes (point 1), et d'études de cas (point 4), le deuxième point, la modélisation, étant le plus souvent réduit ou même absent. Notre recherche porte une attention toute particulière à la modélisation ainsi qu'aux aides à la mise en œuvre. 


\section{La nécessité d'enrichir la sémantique des interfaces conceptuelles entre les concepteurs et autres parties prenantes}

Soutenir la valeur de démocratie implique que les méthodes proposées aident au dialogue entre les concepteurs et les autres parties prenantes (dont nous rappelons qu'elles sont également responsables des valeurs incluses dans les SI). Dans le processus de conception d'un SI, certaines phases requièrent plus que d'autres la coopération de toutes les parties prenantes, et sont ainsi plus propices à un tel dialogue, en particulier les phases de définition des exigences, de tests, et de mise en place du SI. Notre recherche se centre sur la première de ces phases, l'ingénierie des exigences.

L'ingénierie des exigences a pour objectif de déterminer les fonctionnalités du futur système et les contraintes qu'il doit respecter, mais aussi de resituer la visée globale du système par rapport aux finalités générales de l'organisation. L'étude de cette visée et de ces finalités est l'objet de la définition des besoins initiaux (early requirements), phase malheureusement encore mal outillée aux niveaux conceptuel et méthodologique (Regev, 2003). Cette phase est pourtant essentielle au regard de la responsabilité économique et sociale du concepteur, car elle va fixer le niveau macro évoqué plus haut (les paradigmes, les visions du monde sur l'organisation, ses acteurs, ses objets, etc.), qui constituera le cadre dans lequel seront inscrites les fonctionnalités, puis les caractéristiques techniques, du SI.

Dans une perspective de respect de la valeur de démocratie, notre contribution a pour but de faciliter la co-construction des besoins initiaux entre les différentes parties prenantes, ou, a minima, de permettre que les choix réalisés à ce niveau et leurs conséquences sur le système soient explicités. La voie choisie est celle d'un enrichissement des interfaces conceptuelles entre les concepteurs et les autres parties prenantes, pour limiter au mieux les choix implicites. En effet, ces derniers, qui peuvent concerner tous les niveaux (du macro au micro en passant par tous les intermédiaires) sont une des sources de l'intégration «automatique» dans les SI de valeurs, sans que celles-ci n'aient fait l'objet de débats.

En conclusion de cette section, nous pourrions dire que notre objectif est d'outiller la capacité d'agir (la responsabilité) du concepteur de SI (en collaboration avec les autres parties prenantes) pendant l'élaboration du SI, afin que le SI soutienne à son tour la capacité d'agir des parties prenantes dans leurs activités au sein de l'organisation. Notre travail s'inscrit ainsi dans une ingénierie de la responsabilité. 


\section{CONTRIBUTION À UNE INGÉNIERIE DE LA RESPONSABILITÉ}

Cette partie présente un modèle utilisé pour assister la définition des besoins initiaux dans une perspective de démocratie. Ce modèle permet de révéler la chaîne qui va des représentations (paradigme, niveau ontologique, niveau macro) aux normes (niveau micro), en passant par un niveau intermédiaire (les principes). Il est utilisable dans deux configurations : pour analyser une situation existante, et/ou pour construire une alternative. Il peut ainsi aider à comprendre comment certaines valeurs sont intégrées dans les SI, et il peut également aider à promouvoir d'autres valeurs, en assurant leur expression à chacun des trois niveaux.

Cet outil méthodologique est l'un des composants d'une méthode complète d'ingénierie des exigences pour la conception de SIAD, qui prend en compte la responsabilité économique et sociale du concepteur. Pour le détail de cette méthode, on se reportera à Salles (2013). Le contenu du modèle est d'abord présenté, puis une application du modèle est proposée en illustration.

\section{Le modèle Doxai, Principes, Normes (DPN)}

Ce modèle distingue et explicite trois niveaux dans l'expression des valeurs : le niveau des doxai, celui des principes et celui des normes (voir figure suivante).

Figure 1 - Schéma global du modèle DPN

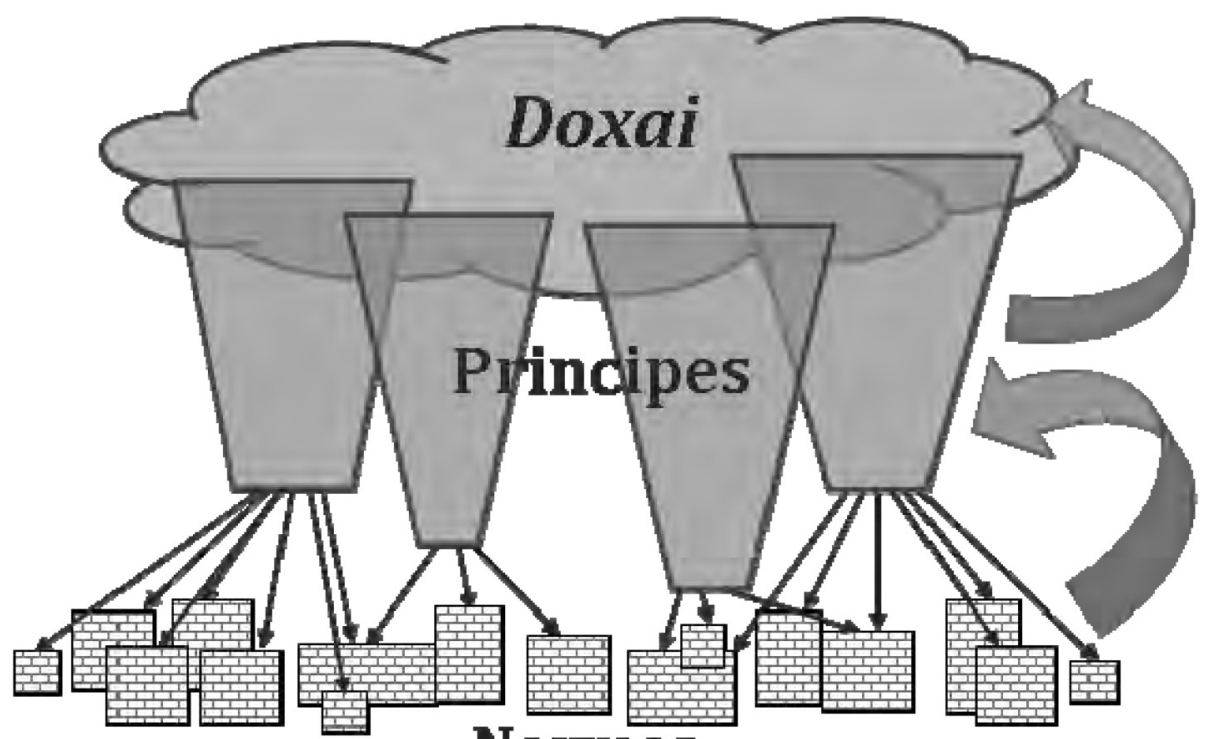

Normes 


\section{Les doxai}

Le premier niveau, celui des doxai, renvoie aux visions du monde qui règnent dans l'organisation (niveau macro) : statut ontologique des grands objets impliqués dans l'organisation, choix paradigmatiques, grandes options stratégiques ou politiques. Les options prises à ce niveau (le plus souvent de façon implicite, voire tacite) vont prescrire le sens qui sera incarné dans les SI, et, notamment, les grandes valeurs éthiques qui y seront ou non respectées.

Dans la deuxième partie de cette partie nous présenterons un exemple d'application du modèle DPN, incluant la description de deux doxai. Nous nous limiterons donc ici à donner trois exemples. Le premier exemple est celui des grandes doxai sur l'économique et le social. Une doxa dissocie l'économique et le social, quand une autre doxa les considère absolument indissociables. Notons que notre travail s'inscrit résolument dans cette seconde doxa, et traite de la responsabilité économique et sociale comme d'un tout non décomposable.

Un deuxième exemple est celui de l'entreprise. Celle-ci peut être considérée, comme le fait la théorie de l'actionnaire évoquée plus haut comme un système dont la raison d'être est de produire de la valeur actionnariale (on pourrait aujourd'hui ajouter : le plus rapidement possible), elle est alors vue comme un actif liquide, dont la pérennité n'est pas spécialement recherchée. Une autre représentation de l'entreprise peut la concevoir comme un système dont la mission est de produire des biens et des services.

Le troisième exemple concerne les représentations du territoire (par exemple pour une collectivité territoriale). Une première doxa considère le territoire comme donné (postulé), il est alors vu comme un espace fini, délimité par des frontières. Une seconde doxa considère le territoire comme devant être créé à la faveur d'un projet coordonnant des acteurs proches spatialement, projet et territoire étant alors indissociables (un territoire ne pouvant exister qu'au travers d'une suite de projets).

\section{Les principes}

Les doxai sont des visions générales, qui ne peuvent être utilisées pour organiser et contrôler les activités de l'organisation. Des dispositifs plus structurés sont nécessaires.

Les principes vont préciser à l'aide de quels concepts, quels objets et quelles méthodes les orientations du niveau précédent vont être rendues plus opérationnelles. Les principes structurent les organisations (de toutes tailles) et leurs actions. Comme on le verra, les principes engendrent les normes. Nous les qualifions de machines à produire des normes (Salles, 2013). 
On retrouve à ce niveau :

- le mode de définition de la mission et du périmètre de l'organisation considérée,

- les objectifs globaux (non chiffrés) assignés à l'organisation,

- les modes de traitement des parties prenantes dans un projet (mode de détermination de la liste des parties prenantes, de leur hiérarchie, de leurs attributions...),

- les modes de management : modes de prises de décisions, modes d'organisation globale, modes d'organisation des processus, types de relations avec des acteurs extérieurs à l'organisation, principes régissant les règles internes, etc.,

- la logique de la comptabilité analytique,

- la logique des critères de sélection des investissements,

- les méthodes de définition des indicateurs d'évaluation, etc.

Ces principes s'incarnent dans les SI :

- définition des missions du SI, de son périmètre du SI (incluant ou non des acteurs externes à l'organisation),

- modes de conception et de gouvernance des SI,

- structures des SI : modèles conceptuels de données, de traitements et d'interfaces,

- principes de répartition des décisions et des actions entre l'utilisateur et l'application,

- méthodes pour le calcul des indicateurs (dont conventions de quantification), leur interprétation, la définition des seuils de déclenchement automatique d'actions,

- principe de contrôle en temps réel des activités des salariés, etc.

Notons que dans la littérature le niveau des principes est rarement évoqué, étant confondu avec le niveau suivant (celui des normes). Or, il représente selon nous le lieu majeur de l'expression de la démocratie, ou de son empêchement. Plus largement, c'est essentiellement à ce niveau que se produit l'intégration des valeurs éthiques (ou " anti-éthiques ») dans les SI.

Les représentations évoquées plus haut pour l'entreprise et le territoire vont donner lieu à des principes différents selon la doxa adoptée, conduisant à une prise en compte différente de la valeur de démocratie.

Dans la première représentation de l'entreprise, la structure du SI reflète une décomposition de l'organisation en centres de profit. La comptabilité analytique est organisée selon la logique du résultat (coûts, marge). L’accent 
est globalement mis sur l'information financière, les ratios financiers, l'évolution de la valeur actionnariale. La conception et la gouvernance des SI impliquent un nombre très réduit de parties prenantes, dont les rôles sont fortement contraints.

Dans la seconde représentation, le SI est un lieu de consolidation / mémorisation des connaissances. Il doit aider à identifier l'évolution des besoins fondamentaux. La comptabilité analytique est organisée autour de la production de valeur ajoutée (Brodier, 2013). Dans la conception des SI et leur gouvernance, les différentes parties prenantes disposent de droits comparables.

Au niveau de politiques de développement économique, les deux visions du territoire induisent des logiques d'action totalement différentes, voire opposées (Salles, 2010). L'action politique sera centrée sur l'entreprise pour la première doxa (qui considère le territoire comme donné, doté de ressources finies), sur les interrelations entre acteurs pour la seconde (pour laquelle le territoire est créé à la faveur de projets, et pour laquelle il convient donc de densifier ces relations). Le SI exprimera l'une ou l'autre de ces logiques, par le biais des entités considérées dans les modèles conceptuels. Dans le cas de la première représentation du territoire, il est possible que le SI n'inclue dans ses modèles conceptuels aucune information sur les relations entre acteurs, rendant tout changement de politique difficile voire impossible.

\section{Les normes}

Nous désignons par norme tout ce qui encadre concrètement l'activité des personnes au sein des organisations. C'est le niveau de l'expression tangible des valeurs éthiques (ou de leur absence). Il est produit par l'application des principes, modèles et méthodes du niveau précédent :

- les modes de managements vont se traduire, par exemple, par une organisation du temps de travail précise (horaires flexibles, temps lissé sur l'année, en $3 / 8 \ldots$ ), des procédures de gestion, des processus de décision concrets (liste des types de participants à la prise de décision, informations à recueillir, délai, évaluation...), la liste des comptes de la comptabilité analytique, etc.,

- ces normes vont être codées dans les SI : logiciels qui intègrent l'organisation du travail et les procédures, logiciels de contrôle de l'activité en temps réel, tableaux de bord rassemblant des indicateurs chiffrés, listes de critères (de classement, de tri...), etc.

Ce troisième niveau (niveau micro) est le plus visible, et par suite, il est souvent le seul pris en compte dans les démarches d'éthique informatique. 
Au niveau des normes, le SI de l'entreprise considérée selon la première doxa produira des ratios financiers, des indicateurs de mesure des coûts, des logiciels encadrant les salariés à l'aide de procédures contraignantes (toutes destinées à réduire les coûts), etc. Dans la seconde représentation, le SI calculera la valeur ajoutée disponible, mesurera les externalités positives (innovation...) et négatives (pollution...), etc.

Le SI au service d'une politique de développement économique territorial inclura par exemple des critères de sélection des dossiers de demandes de financement différents selon les deux visions du territoire. Dans le premier cas, le périmètre de ces critères sera celui de l'entreprise, dans le second, il sera étendu aux regroupements d'entreprises et d'autres acteurs.

\section{Application du modèle DPN à l'évaluation}

Le modèle brièvement présenté plus haut peut être appliqué à plusieurs types d'objets et de concepts qui interviennent dans la vie des organisations. Afin d'aider à la mise en œuvre de la méthode qui a été évoquée plus haut, plusieurs entités ont fait l'objet de modélisations DPN doubles. La représentation dominante de l'objet a été modélisée, puis une représentation alternative a été construite. L'entreprise (Salles, Colletis, 2013), le travail, le territoire, les ressources d'un territoire,... ont ainsi été modélisés aux trois niveaux de DPN, selon la doxa dominante, puis selon une vision alternative.

Nous allons ici brièvement illustrer l'utilisation du modèle DPN à propos de l'évaluation des salariés au sein des organisations (publiques ou privées), en présentant deux représentations concurrentes de cette catégorie.

\section{Deux doxai de l'évaluation}

Pour une première doxa l'intention de l'évaluation est de vérifier une conformité à une cible ou un modèle prédéfini. Cette doxa considère les différences comme des frottements à éliminer, et vise l'homogénéité. Elle estime que tout peut être quantifié et comparé, et que, le réel existant de façon immanente, la quantification quantifie ce qui existe (le réel) de façon objective, neutre, technique. Ainsi, la quantification ne saurait être débattue (notons que ceci s'oppose à la valeur de démocratie). L'activité de manager consistera pour l'essentiel à comparer, classer, sélectionner, récompenser/punir (et surveiller). Cette doxa, basée sur le contrôle (et non la confiance) renvoie à la figure de l'homo oeconomicus (calculateur, égoïste) et promeut la compétition comme seul mode de rapport entre les hommes, mais aussi les organisations, les territoires, les états... (Metzger, 2010). 
Il convient de noter que cette doxa, née au sein des entreprises privées, s'est étendue aujourd'hui au secteur associatif, aux institutions (dont l'université), aux services publics, aux États. Le Galès (2005), évoquant l'évaluation, par l'État, des politiques des collectivités territoriales au Royaume-Uni, note ainsi que «en termes de rapport à la société, cette théorisation implicite n'est pas bâtie sur des dynamiques de mobilisation, de coopération, mais de contraintes d'un modèle optimum de gestion dont les fins de sont jamais explicitées ni, à plus forte raison, discutées ».

Dans une deuxième représentation de l'évaluation, l'intention est d'identifier des compétences (ou leur absence), des savoirs, dans le but de les développer, les promouvoir. Cette doxa considère que tout n'est pas réductible à des valeurs numériques (de même que toute valeur n'est pas assimilable à une valeur monétaire), et que tout n'est pas comparable (notion d'incomparabilité). La réalité est vue comme une construction, et sa quantification suppose des conventions d'équivalence préalables (Desrosières, 2003). L'évaluation peut être autre que quantitative. Manager sera alors identifier des différences, rechercher des complémentarités, des latences, des émergences, se projeter dans l'avenir. Cette représentation de l'évaluation reconnaît la diversité comme seule source d'évolution. Elle est fondée sur la confiance (dans les capacités de tous et de chacun), celle-ci étant vue comme un élément indispensable de développement d'une organisation quelle qu'elle soit. Cette deuxième doxa est plus propice au respect de la valeur de démocratie.

\section{Quelques principes qui incarnent les deux visions de l'évaluation}

Les principes étant en grand nombre, nous n'en donnons ici que quelques exemples. Au niveau des principes de management, la première doxa organise une évaluation atomisée (et non systémique), pour les salariés (évaluation individuelle), et pour les coûts (les éléments de coûts sont considérés comme indépendants les uns des autres). Les types d'indicateurs sont décidés par le seul management (et, parfois, par le seul haut management). Il en est de même pour la définition des valeurs limites, des interprétations-types... Le SI intègre ces principes, en définissant les structures de données, les programmes permettant la collecte automatique des données, le calcul automatique des indicateurs et des interprétations-types, et le déclenchement automatique d'actions à partir de certaines valeurs.

La mise en œuvre de tels principes peut s'opposer au respect de valeurs éthiques. Le Galès (2005) étudiant le cas des institutions publiques R.U., observe que « toute dépense, tout programme doit être engagé à l'aune du rapport coût/efficacité sans autre critère, ce qui requiert la production d'indicateurs, de mesures de performance, d'évaluation, de mise en concurrence ", et remarque 
que ces principes sont en contradiction avec des principes existant auparavant, et qui, pour des marchés publics, sélectionnaient des entreprises sur des critères incluant des aspects sociaux, moraux ou politiques (des entreprises travaillant avec l'Afrique du Sud au temps de l'apartheid étaient ainsi écartées des marchés de certaines collectivités). Cette tendance à limiter drastiquement le nombre de critères de sélection ou d'indicateurs d'évaluation, aboutissant à un critère ou un indicateur unique, existe également dans les entreprises.

Dans la deuxième représentation de l'évaluation, l'organisation est considérée comme un système (et non comme une somme de parties). L'évaluation est appliquée à des collectifs, et elle est faite en dynamique (et mesure donc les évolutions). Les modes d'évaluations, quantitatifs ou qualitatifs, sont débattus par les parties prenantes concernées. Le SI rend compte d'une cartographie des compétences et de son évolution dans le temps. La conception des logiciels de collecte de données, de calcul d'indicateurs, de constitution de tableaux de bord, etc., inclut les parties prenantes.

\section{Quelques exemples de normes}

Les normes vont rendre les principes d'évaluation opérationnels. Les normes issues de la première représentation vont ainsi par exemple produire des notations des salariés, des entreprises, des États, mais aussi la sélection de tel ou tel investissement, de tel ou tel salarié, ou encore la décision de lancer ou non tel ou tel projet. Lorrain (2005) note ainsi que «le taux d'actualisation et sa durée choisis pour un grand ouvrage vont le rendre réalisable ou non ", ou que "les durées d'amortissement se traduisent en bout de chaîne par des tarifs payés par les usagers".

On remarquera que les indicateurs, qui constituent la norme par excellence, entraînent des effets performatifs notables. En accord la maxime "Dites-moi comment vous allez m'évaluer et je vais vous dire comment je vais me comporter $»^{19}$, on observe de nombreux cas où l'activité n'est plus guidée par l'objectif à atteindre ou la mission à réaliser mais par le seul but d'améliorer les indicateurs d'évaluation.

Les normes de la seconde représentation produisent des résultats d'évaluation non systématiquement quantitatifs, à des critères de sélection à visées multiples, éventuellement complétés par la discussion pour aboutir à un choix final.

19. "Tell me how you will measure me and I'll tell you how I will behave". 


\section{CONCLUSION}

Dans ce texte, nous avons voulu apporter une contribution à la prise en compte de la responsabilité économique et sociale (ces deux dimensions étant indissociables) des concepteurs de SI. Cette responsabilité s'inscrit plus largement dans la responsabilité morale des concepteurs de SI, et relève ainsi de l'éthique informatique. Celle-ci est un domaine fortement pluridisciplinaire qui est encore en formation. Nous avons proposé une revue de littérature, et relevé un ensemble de questions qui nous paraissent essentielles pour les développements futurs de ce champ, et, avant tout, pour l'opérationnalisation des résultats de recherche.

Répondant aux observations des philosophes de l'éthique informatique, qui reprochent aux chercheurs en SI de ne considérer que le niveau micro sans s'interroger sur ce qui le détermine au plan ontologique, nous avons ensuite exposé notre positionnement. Ce dernier est celui d'une recherche critique, qui reconnaît dans les SI des dispositifs de pouvoir, et vise à soutenir au sein des organisations une valeur éthique primordiale : la démocratie. La voie choisie pour ce faire s'attache à révéler les visions du monde (niveau macro, ontologique) qui s'incarnent dans les SI, afin, ensuite, de promouvoir une réelle diversité des paradigmes.

Un outil méthodologique a été présenté et illustré, le modèle DPN, qui décrit la chaîne qui va des grandes représentations (désignées ici comme des doxai), jusqu'aux normes qui prescrivent les activités des salariés et des partenaires de l'organisation, en passant par le niveau intermédiaire des principes. Ces derniers, maillon essentiel du passage d'une représentation générale à sa traduction opérationnelle, concrète et contraignante, sont peu considérés dans la recherche du domaine.

La généralisation des SI à toutes les organisations et à toutes leurs activités, et singulièrement à la prise de décision, qui accompagne une orientation financiarisée de l'économie, soulève des problèmes éthiques de plus en plus nombreux et de plus en plus aigus. On peut donc s'inquiéter de ce que l'éthique informatique refuse (pour l'essentiel) de reconnaître dans les SI des instruments de pouvoir. Le risque est ainsi qu'elle puisse, malgré elle, servir de prétexte pour délégitimer une pensée politique ou même citoyenne. 


\section{RÉFÉRENCES BIBLIOGRAPHIQUES}

BARQUIN, R. (1992), In Pursuit of a Ten Commandments for Computer Ethics, Computer Ethics Institute.

BELL, F., ADAM, A. (2004), The Problem of Integrating Ethics into IS Practice, Proceedings of the 13th European Conference on Information Systems (ECIS), Finland, 189-199.

BOLLECKER, M., MATHIEU, P., CLEMENTZ, C. (2008), L'évolution des systèmes de gestion face aux enjeux de la responsabilité sociale : le cas de la comptabilité et du contrôle de gestion, Gestion 2000, 4, 49-65.

BORNING, A., WADDELL, P., FORSTER, R. (2007), UrbanSim: Using Simulation to Inform Public Deliberation and Decision-Making, in Chen, H., Brandt, L., Gregg, V., Traunmuller, R., McIntosh, A., Dawes, S., Hovy, E., Larson, C. A. (eds), Digital Government: E-Government Research, Case Studies, and Implementation, Springer-Verlag, 439-463.

BREY, P. (2008), Values in Technology and Disclosive Computer Ethics, in Floridi, L. (ed.), The Cambridge Handbook of Information and Computer Ethics, Cambridge, Cambridge University Press, 41-58.

BRODIER, P.-L. (2013), La logique de la valeur ajoutée, une autre façon de compter, L'Expansion Management Review, 148, 20-27

BULL, C. (2009), A Review of Ethical Theory in the 'Upper Echelons' of Information Systems Research, Proceedings of the 17th European Conference on Information Systems, Verona, Italy.

BYNUM, T. W. (1985), Computers and Ethics, Metaphilosophy, 16(4), 263-377.

BYNUM, T. W. (2011), Computer and Information Ethics, in Zalta, E. N. (ed.), The Stanford Encyclopedia of Philosophy, The Metaphysics Research Lab, Stanford University.

CAPRON, M., QUAIREL-LANOIZELÉE, F. (2007), La responsabilité sociale d'entreprise, Paris, La Découverte.

CHAE, B., PARAdiCE, D., COURTNey, J. F., CAROL, J. CAGLE, C. J. (2005), Incorporating an Ethical Perspective into Problem Formulation: Implications for Decision Support Systems Design, Decision Support Systems, 40, 197-212.

CIGREF (2009), Systèmes d'information éco-responsables. http://www.cigref.fr/cigref_ publications/RapportsContainer/Parus2009/Systemes_d_information_eco-responsables_ CIGREF_2009.pdf, consulté le [26 septembre 2013].

CIGREF (2013), Identification et gouvernance des enjeux éthiques émergents dans les systèmes d'information (IDEGOV). http://www.fondation-cigref.org/wp/wp-content/ uploads/2013/03/Les-Essentiels-IDEGOV-synthese.pdf, consulté le [26 septembre 2013].

COTTONE, R. R., CLAUS, R. E. (2000), Ethical Decision-Making Models: A Review of the Literature, Journal of Counselling Eु Development, 78, 275-283.

COTTONE, R. R. (2001), A Social Constructivism Model of Ethical Decision-Making in Counseling, Journal of Counselling Eु Development, 79, 31-45.

DESROSIÈRES, A. (2003), Du réalisme des objets de la comptabilité nationale, Congrès de l'Association Française de Sciences Économiques, septembre, Paris.

DONALDSON, T. J. (1982), Corporations and Morality, Prentice-Hall, Englewood Cliffs, NJ. FLORIDI, L. (2008), Foundations of Information Ethics, in Himma, K. E., Tavani, H. T. (eds.), The Handbook of Information and Computer Ethics, John Wiley \& Sons, Hoboken, 3-23. FLORIDI, L. (2010), Preface, in Floridi, L. (ed.), The Cambridge Handbook of Information and Computer Ethics, Cambridge, Cambridge University Press, ix-xv. 
FRIEDMAN, B., KAHN, P. H., BORNING, A. (2002), Value Sensitive Design: Theory and Methods, University of Washington, Dept. of Computer Science \& Eng. Technical Report, 02-12-01.

HABERMAS, J. (1999), A Genealogical Analysis of the Cognitive Content of Morality, in Habermas, J. (ed.), The Inclusion of the Other, Cambridge, Polity Press, 3-48.

HALL, R., STAHL, B. (2012), Against Commodification: The University, Cognitive Capitalism and Emergent Technologies, tripleC, 10(2), 184-202.

JOHNSON, D. (1985), Computer Ethics, First Edition, Englewood Cliffs, Prentice-Hall.

KVASNY, L., RICHARDSON, H., (2006), Critical Research in Information Systems: Looking Forward, Looking Back, Information Technology EB People, 19(3), 196-202.

LE GALÈS, P. (2005), Contrôle et surveillance : la restructuration de l'État en GrandeBretagne, Lascoumes, P., Le Galès, P. (eds), Gouverner par les instruments, Presses de Sciences Po « Académique », Paris, 237-271.

LASCOUMES, P., LE GALÈS, P. (2005), Conclusion, in Lascoumes, P., Le Galès, P. (eds), Gouverner par les instruments, Presses de Sciences Po « Académique », Paris, 357-370.

LE MOIGNE, J.-L. (1977), La théorie du système général. Théorie de la modélisation, Paris, Presses Universitaires de France, $4^{\mathrm{e}}$ édition mise à jour 1994.

LORRAIN, D. (2005), Les pilotes invisibles de l'action publique. Le désarroi du politique? in Lascoumes, P., Le Galès, P. (eds), Gouverner par les instruments, Presses de Sciences Po "Académique », Paris, 163-197.

MANER, W. (1996), Unique Ethical Problems in Information Technology, Science and Engineering Ethics, 2(2), 137-154.

MASON, R.O. (1986), Four Ethical Issues of the Information Age, MIS Quarterly, 10(1), $4-12$.

MATHIESON, K. (2007), Towards a Design Science of Ethical Decision Support, Journal of Business Ethics, 76, 269-292.

MAUGERI, S. (2007), Travail, dispositif de gestion et domination, XI ${ }^{\text {èmes }}$ Journées internationales de sociologie du travail, 20/22 juin, Londres.

MEREDITH, R., ARNOTT, D. (2003), On Ethics and Decision Support Systems Development, 7th Pacific Asia Conference on Information Systems, 10-13 July, Adelaide, South Australia.

METZGER, J.-L. (2010), Peut-on sortir de la crise sans re-penser la gestion ?, Savoir agir, 13, 39-47.

MINGERS, J., WALSHAM, G. (2010), Towards Ethical Information Systems: The Contribution of Discourse Ethics, MIS Quarterly, 34(4), 833-854.

MITCHAM, C. (1995), Computers, Information and Ethics: A Review of Issues and Literature, Science and Engineering Ethics, 1(2), 113-132.

MOATI, P. (2008), L'économie des bouquets. Les marchés de solution dans le nouveau capitalisme, Éditions de l'Aube, La Tour d'Aigues.

MOOR, J. H. (1985), What Is Computer Ethics?, Metaphilosophy, 16(4), 266-275.

NISSENBAUM, H. (1998), Values in the Design of Computer Systems, Computers and Society, March, 38-39.

NEUMAN, W. L. (2000), Social Research Methods: Qualitative and Quantitative Approaches, Fourth edition, Allyn and Bacon, Needham Heights, MA. 
REGEV, G. (2003), A Systemic Paradigm for Early IT System Requirements Based on Regulation Principles, Thèse de doctorat, Faculté Informatique et Communications, École Polytechnique Fédérale de Lausanne.

RICCEUR, P. (1998), Éthique, Encyclopædia Universalis.

RICCEUR, P. (2000), De la morale à l'éthique et aux éthiques, in Appel, K. O. (éd.), Un siècle de philosophie 1900-2000, Paris, Gallimard, 103-120.

ROGERSON, S., WECKERT, J., SIMPSON, C. (2000), An Ethical Review of Information Systems Development: The Australian Computer Society's Code of Ethics and SSADM, Information Technology and People, 13(2), 121-136.

ROWE, F. (2009), Les approches critiques en Systèmes d'Information : de la sociologie de la domination à l'éthique de l'émancipation, Économies et Sociétés, 12, 2081-2114.

SALLES, M. (2007), Présentation, "Représentations, modèles et normes pour l'entreprise », Revue Droit et Société, 65, 21-26.

SALLES, M. (2010), Supporting Public Decision Making - a Progressive Approach Aided by an Ontology, International Journal of Decision Support System Technology, special issue: Semantic Decision Support Systems, 2(1), 21-36.

SALLES, M. (2013), Ingénierie de méthodes d'ingénierie des exigences pour l'aide à la décision, Habilitation à diriger des recherches, Université de Toulouse I-Capitole/IRIT.

SALLES, M., COLLETIS, G. (2013), Déconstruire la doxa dominante, construire une pensée politique alternative. Du lien entre les représentations, les principes et les normes, LoSguardo, special issue The Instruments of the Power, XIII(10), 391-414.

SELIGMANN, P. S., WIJERS, G. M., SOL, H. G. (1989), Analysing the Structure of IS Methodologies, An Alternative Approach, Proceedings of the $1^{\text {st }}$ Dutch Conference in Information Systems, Amersfoort, The Netherlands.

SMITH, J., HASNAS, J. (1999), Ethics and Information Systems: The Corporate Domain, MIS Quarterly, 23(1), 109-127.

STAHL, B. C. (2008a), Researching Ethics and Morality in Information Systems: Some Guiding Questions, ICIS 2008 Proceedings.

STAHL, B. C. (2008b), Ethical Issues of Information and Business, in Himma, K. E., Tavani, H. T. (eds), The Handbook of Information and Computer Ethics, John Wiley \& Sons, Hoboken, 311-335.

TURKLE, S. (2004), How Computers Change the Way We Think, The Chronicle of Higher Education, 50(21), B26.

VAN DEN HOVEN, J. (2008), Moral Methodology and Information Technology, in Himma, K. E., Tavani, H. T. (eds), The Handbook of Information and Computer Ethics, John Wiley \& Sons, Hoboken, 49-67.

WALSHAM, G. (1993), Ethical Issues in Information Systems Development, The IFIP 8.2 Working Group Information Systems Development: Human, Social and Organizational Aspects, North-Holland, Noordwijkerhout, The Netherlands.

WALSHAM, G. (2011), Interpreting Information Systems in Organizations.

WALSHAM, G. (2012), Are We Making a Better World with ICTs? Reflections on a Future Agenda for the IS Field, Journal of Information Technology, 27(2), 87-93.

WIENER, N. (1948), Cybernetics or Control and Communication in the Animal and the Machine, Cambridge, MIT Press.

WIENER, N. (1989), The Human Use of Human Beings. Cybernetics and Society, Free association books, London. First published in 1950 and revised in 1954, Houghton Mifflin. 